von do Schicht absorbict wird, wahrend beim Verschwinten diescr Flüssigkeitsverbindung die lichthurchlässige plache den Lichtstrahl gatbz oler teilweise reblektiert und in kichtung des

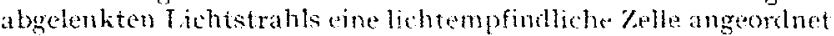

ist, die beim Auftreffen des Lichtstrabls Regel- oler Schaltgerite in Tátigkeit setzt. Fine oder beicle Koudensationsflächen kinnen kugelansschnita. kegel., pyramiden- oder zylinderfirmig atsgebiltet sein.

\title{
B. Erteilte ausländische Patente
}

61 Ital. P. 384486 \%. 24. IV. 11) Haul Kinchalik. Magdaburs. und Gustat dalolt Kienitz. Eberswakle. Reinigung von Zellstoff. Zwecks Entfernung der Hemizeliulosen wird dis nach dem Alkaliverfaliren atufeschlossene Kochgut umittelbar nach Entfernung der Kochlauge im Koeher selbst unter Ausch!ut\} von Juft, $z$. B. indem der kocher unter Vakunm gesetzt wird, mit fricher kalter Kochlange behandelt. Wis sich rabei orwärmende fange wirl dann ansphliebend zum dufschlub verwendet.

61 Dän. P. 59284 v. 13. 1II. 19411, ausg. 8. XII. 19+1. Torsk If ydro-Elektrisk Kvaelstofaktieselskah, (Nolo. Entfernung von basischen Stoffen aus Sulfitzelluloseablauge. Man behandelt dic Abfallatuge: gegebenenfalls nach Entfernung des Zuckers durch Gärung, mit sauren onganixhen basenanstauschern, sogenannten Organolithen. Natrium- oler Kalziumbibindungen enthatende faugen konwen auch mit organdochen Basonanstauschem, die als austauschbare Gruppe dis Ammoniumgruppo 'nthaiten, behandelt werden. Die Regenerierung der gebratheh ten Basenaustauscher erfolgt lurch Behandlung mit wäbriget sctrwefliger Säure.

61 Norweg. P. 64263 \%. 10. V. 19+0, ausg. 15. X11. 1911. Norsk Hydro-blektrisk Kratelotoktieselskab, Oslo. Entfernung der organischen Substanz aus Sulfitzelluloseablauge. Man thitzt die lauge. gerebenentals nach Anreicherung mit schweflige Saure, unter Zusatz einer reringen Henge Selen unter Druck Beispid: Line Kalziumbisulfitablatuge die $12 \%$ Truckenstoffe. $1.06 \%$ Schwefeklioxyd und $1 \%$ halmiumoxyd enthalt, wird

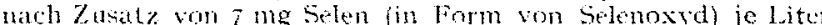

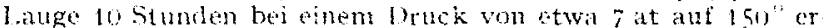
hitzt. Whe ansgefalite organisohe Substanz kann man be

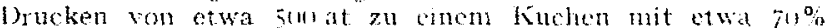
Trockenstoffen verpresien.

61 Ital. P. 383219 v. 20. I11 1939. Cartiere Burgo Soc Au. Verzuolo. Cunco. Vorbereitung von Zellstoff für die Nitrierung.
$100 \mathrm{~kg}$ roher oler gereinigter zellstoff werden 2 Stunden mi 2000 I.iter ence l.ösuner von $35 \mathrm{~kg}$ Natriumbralroxval und $10 \mathrm{k}$ Ferriayankalium (rotes Blutlaugensalz) behandelt, umi zwar zu nachst bei normaler Temperatur, die dann allmählich aut of crhoht wird. Anschlielsend wird mit Wasser gewaschen.

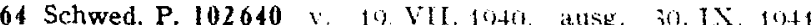

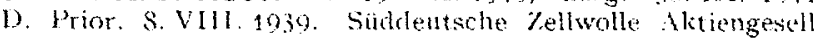
schatt. Kehheim a. d. Donau (Etincler: $E$ Abele). Veredelung von Alkalizellulose. Man läbt die fein zerteilte Alkalizollulose in dünner Schicht über sinen Turm herbrieseln, der aus ab. wechselnd ibercinamier angeordneten konischen und zylindrischen Toilstücken besteht. Die zylindrischen Teilstücke sind nit Offnumgen versehen, durch dic kalte Luft gegen die herabrieselnde 1lkalizellukse geblasen wird, un cine kiuhlung and Temperaluren unterhalb 25 "zu erzielen. Die Is uhbung bewirkt. dab keine Sulfilerming der Alkalizellulose erfolgt

68 Ital. P. 383773 々. 3. IT. 1940. Elino Cariolago und Christiano Rigon, Italien. Gewinnung von Ligninstoffen aus alkalischer Zelluloseablauge. Nach Ventrahisierme der I auge wird mitiels Salzsaure und Aluminiumsulfat gefalli. Das ausgefällte Lignin wirl nach Mischung nit lrockenblut zu plastischen Masien verprebt.

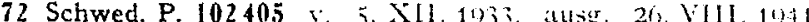
R. Falck. Jerusalem. Holzimprägnierung, Frisch gefallto Holz stamme verden von den Stirnflächen atu durch möglichst

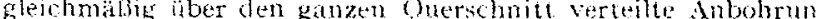
gen unter Druck mit konzentrierton wabrigen I.dsangen von Holzsehutzmittem imprägniert. Geeignete Losungen sind z. B. 10... 20proz. Zinkchlorid- oder kupfersulfatlosung 20... 31)proz. Entimoniluorid, Annoniumflaorik-, Zinkfluorsilikatoder Magnesiumfluorsilikatlösung, $10 \ldots$ 20proz. Natrium. arsenit-oher Natriumarsonatlisumg, 1\&...21)proz. Natrium. bichromatlonmen

\section{Deutsche Patentanmeldungen.}

Die einleitenden Zahlen und Buchstaben vorn links bezeichnen die Klasse und dic Gruppe, die Zahl hinter dem schrägen Strich ist die der Dezimalgruppennummer. Darauf folgt das Aktenzeichen. Im Fall der Inanspruchnahme einer ausländischen Priorität ist am SchluQ Iand und Tas der Voranmeldung. im Fall der Inanspruchnahme der Priorität der Schaustellung au' einer Ausstellung ist am Schlub diese und der Tag ihrer Eröfnung angegeben. Dic Nennung des Erfinders erfolgt aui Grund von Angaben des Anmelders. Für die angegebenen Gegenstände haben die Genannten an dem bezeichneten Tage die is: teilung eines Patentes nachgesucht. The Anmeldungen werden gemäB $\$ 30$ Absatz 3 PG. vom Tage dieser Bekanntmachung ab drei Monate lang in der Auslegehalle des Reichspatentamts zur Einsicht iür jedermanu ausgelegt. Der Gegenstand der Anmeldung ist einstweilen gegen unhefugte Benutzung geschützt.

Pateritblat Nr. 39 rom 24. 1 X. 1942.

$38 \mathrm{c}$, 19n. I 6f664. Fri.; Adolf Mengen, Krefeld, Eugen lock

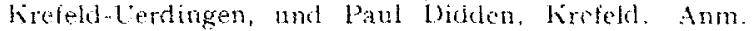
L. C. Farbenindustrie A.-Ci.. Fankfurt a. M. Verfahren zum Verkleben von Massivbolz mit hohem Feuchtigheitsgehall. 19. V. 1939. Protektorat Böhmen uml Wahren. latentblatt $\mathrm{Nr}$. fo rom 1. X. 1042

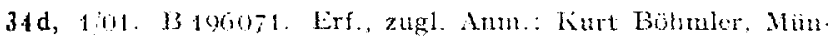
chen. Holzspaltmesser zum Zerkleinern son Tolzscheiter zu Brennholz: Zus. z. Patent 6726s9. 7. XT. 1941 38f, 3jo2. Frf, zugl. Anm : Gerlard Homey, Wittlich, Bez. Trier. Vertiaten zur Herstellung von zylinirischen Holzgrfalen. 28.1938.

55c, 1. V 34874. Erf: Diph-Ing. Hermann Breminger, Heidenkeim, Brenz. Anm, Fitma 1. It Voith, Heidenheim, Brem Verfahren zum fortaufemen Bleichen von Fiserstofen, inshesontere ron Zellstoff. 16. 1. 1938.

89i, 102. S128310. Erf., zugl. Anm1.: Max Seidel, MinchenSolli. Vertahren zur tbextragung von Warme: heiBer Holzzuckerwïzen. 6. VIIT. 1937. Osterreich.

\section{Schrifttumsberichte.}

\section{Allgemeines.}

03 Die Entwicklung der sowjetrussischen Holzverarbeitungs-

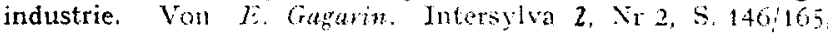
$10+2$.

Da dit: Hauptmasse ler russischen Holzvorrate in wh. erschossenen nordlichen (rebieten stockr, wird die lintwicklung holaverabeitenler Industrien dort fast immir zu cinen vielseitigen Problem. Schon ror der russischen Revolution ist die Erschließung der nördlichen Waldmassive in die Wege geleitet worden. Ihre tatsabhliche Verwirklichung steht jedoch noch aus. Dic sorliegencio arbeit beschäfigt sich mit lieser Entwicklung und erortert solann den Niedergang der russischen Holzindustrie währem der ersten Jahre nach der Revolution und anschliebend ihren dufschwung im Zusammenhang mit der etwa 1927 in Gang gekommenen sog. "Inclustrialisierung" des landes. Fs wid gereigh, wie der forst- und Holawirtschal ane nur antergeordnete kolle in ker Gesamtwirtschaft Rub. lands zugedacht wa:, wie sie nur dazu benulat wurde, Derisen anzubringen. Diese Sachverhalte sowie die planiose Anlage und Verteilung never Betriebe zusammen mit de Vernachlassigung des Transportwesns fühten zu einer gefahrlichen Krise. In den letzton Jahren machten sich jedoch lestrebungen $z u$ cinem planvollen Ausbau cler forstwirtschaft in SowjetruBland bemerkbar. Hierzu werden im zweiten Teil ler Ablandlung zahlenmabige Angaben über Verteilung, Ausribtung und Ixistung der russischen Holz- und Fapierindustric gegeben. Im Vergleich zur Forkriegszeit hat sich die Jizeugung der Holawirtschaft veroleifacht, die der Papierindustrie ist um das fiunffache gestiegen. Fine weitere Steigerung in clor Holzverarbeitungsindustrie, ohne planmabige Erschliebung nowor Waldgebiete, wird fïr unmogglich gelialten; in der nächsten 